\title{
Modern trends in applied probability: part 1
}

\section{A collection of two overview papers}

\author{
Ross McVinish ${ }^{1} \cdot$ Yoni Nazarathy ${ }^{1} \cdot$ Giang Nguyen $^{2}$ \\ Published online: 9 October 2020 \\ (c) Springer Science+Business Media, LLC, part of Springer Nature 2020
}

This is the first part of a special issue comprising surveys on modern trends in applied probability. The special issue arose out of the 20th INFORMS-APS conference. This biannual conference was held in Brisbane Australia on July 3-5, 2019, and the next edition is scheduled to be run in (or from) Nancy, France, in July 2021. The 20th edition of the conference was attended by more than 250 delegates from around the world and included plenary talks, tutorials, and eight parallel streams of sessions. These parallel streams included both invited and contributed talks. Plenary speakers included Ton Dieker, who presented new work on computational stochastic network analysis, and Nelly Litvak, who presented work on large random networks. A tutorial on approximately reversible stochastic processing networks was presented by Devavrat Shah. A second tutorial dealing with product forms of a broad class of systems was jointly presented by Kristy Gardner and Rhonda Righter. Invited and contributed talks are too numerous to mention; however, the complete book of abstracts is available here:

\section{https://informs-aps.smp.uq.edu.au/full-program.pdf}

Following the conference, a call to prepare survey papers on modern trends in applied probability was made to the delegates. It was followed by a process which involved submission of an expression of interest and open peer review between survey authors, as well as blind external referees. A total of eight expressions of interests were submitted, and we are now happy to present two of these surveys which came to fruition. The first paper is by Kristy Gardner and Rhonda Righter. Expanding on their conference tutorial, they survey product form results for queueing systems with flexible jobs and servers. The second paper is by Jinsheng Chen, Jing Dong, and Pengyi Shi and deals with skill-based routing in service operations management. It is also related to several of the invited talks that the authors presented in the conference.

Yoni Nazarathy

y.nazarathy@uq.edu.au

1 The University of Queensland, St Lucia, Australia

2 The University of Adelaide, Adelaide, Australia 
Both of these surveys treat queueing systems that serve multiple types of customers with multiple types of servers. Indeed, analysis of such queueing systems has attracted much attention in recent years due to both its applicability to service systems and the mathematical elegance that is often associated with the analysis. The first survey presents a state-of-the-art view of what can be done for such systems in terms of explicit product form solutions. The second survey presents a more general viewpoint, dealing with asymptotics, approximations, and choices of scheduling policies. Both play an important part in the world of applied probability, and we hope that you find both surveys insightful.

Guest Editors: Ross McVinish, Yoni Nazarathy, and Giang Nguyen.

Publisher's Note Springer Nature remains neutral with regard to jurisdictional claims in published maps and institutional affiliations. 